\title{
Robust PID Controllers Optimized by PSO Algorithm for Power Converters
}

\author{
Lucas C. Borin, Everson Mattos, Caio R. D. Osorio, Gustavo G. Koch, Vinicius F. Montagner \\ Universidade Federal de Santa Maria \\ Santa Maria - RS, Brasil - 97105-900 \\ lukascielo@gmail.com
}

\begin{abstract}
This paper provides an automated design to provide robust PIDs with fixed control gains, suitable to be applied in power converters whose parameters belong to real intervals. Differently from conventional PIDs which use only a nominal model to obtain the fixed control gains and, a posteriori, verify robustness, the proposed approach ensures, a priori (i.e. during the design stage), robust performance for a set of plant parameters. To illustratethe proposed procedure, two conventional PID controllers are given, to achieve phase margin and crossover frequency for a nominal model of a buck converter. An objective function based on frequency domain specifications is proposed. A particle swarm optimization algorithm is then used to find PIDs, in a large search space that include stable and unstable controllers, allowing to optimize this function for all cases of combinations of plant parameters. A case study for the buck converter illustrates the improvements of performance with the proposed method when compared to the conventional PID controllers. Additionally, the design is used in a more challenging application, for a buck-boost converter suitable for small satellites application, becoming a simple alternative for benchmarks for robust control of power converters.
\end{abstract}

Index Terms-DC-DC converter, Particle swarm optimization, Proportional-integral-derivative control, Robust control.

\section{INTRODUCTION}

Proportional-integral-derivative (PID) controllers are recognizedly important in industry applications. They can ensure, with a simple control structure, for several plants of practical interest, with suitable transient, steady state responses and good margins of stability [1]-[6]. PIDs can be designed from the well-known Ziegler-Nichols methods to the more advanced techniques as fuzzy and adaptive controllers [7][13]. In particular, these advanced control techniques allow to improve the performance, at the price of a more complex control implementation, when compared to simple fixed gains PIDs.

In the literature, there are several works that use PID controllers in power converters and some guidelines for the PID design can be found in [14], based on frequency domain features. The simpler choice for PID, is the fixed gain controller. In this direction, for output voltage control of buck

This study was financed in part by the Coordenação de Aperfeiçoamento de Pessoal de Nível Superior - Brasil (CAPES/PROEX) - Finance code 001. The authors would also like to thank the INCT-GD and the finance agencies $(\mathrm{CNP}$ 465640/2014-1, CNPq Projeto 309536/2018-9, CAPES 23038.000776/201754 and FAPERGS 17/2551-0000517-1). and boost converters, one has, for instance, the works in [15][19]. Other paradigm is the PID gain variable controllers. In this direction, one can cite, for instance, for output voltage control of buck and boost converters, the works [10], [20], [21], that use adaptive or fuzzy method for PID control. Note that adaptive or fuzzy controllers are best indicated in the scenario of unpredictable changes in system dynamics. However, there are situations where controllers with fixed gains are appropriate, such as in constant but unknown system dynamics [2].

In the context of PID fixed control gains, finding suitable gains is a challenge in the controller design, and the difficulty increases with the need to meet multiple control objectives. Moreover, structured parameter uncertainty leads to a set of plants to describe the control system and to the need of design of PIDs that can guarantee limits of performance for this whole set. In this scenario, metaheuristics such as genetic algorithms (GA) and particle swarm optimization (PSO) can be useful, as indicated in [22]. It is important to mention that GA and PSO algorithms have already been used in the design of PIDs in [23]-[25]. For instance, in [23] a PID control based on a multiobjective GA is proposed for a linear brushless DC motor, taking into account uncertainties, with an objective function based on the rise time, overshoot and steady-state error. In [24], a PSO is used to determine the gains of an optimal PID for an automatic voltage regulator system, and a comparison with a GA method is presented in this case. In [25], a PSO is used to find optimum values of the gains of a PID controller in order to reduce the current to the powerassisted steering in electric vehicles, having the objective of minimizing a mean square error function. As remarked in [22], the majority of the publication with metaheuristics in power electronics is on power quality and circuitry optimization. There is a lack of publications based on metaheuristics for control tuning in power converters. Recently, a few works are published using PSO to tuning a PID controller in [26]-[29], but not addressing the design of robust controllers. This makes the further investigation in this direction an important issue.

This paper provides as main contribution a procedure, based on PSO, to obtain fixed gains of robust PID controllers applied for voltage regulation of DC-DC converter with parameters not precisely known, but lying on uncertain intervals. The models of the plants used here contain interval uncertain parameters, 
leading to a set of linear models for each of the situations of extreme values of these uncertain parameters. As a first case study, two conventional PID designs were performed, for a buck converter, relying only on a nominal plant to meet phase margin and crossover frequency specifications, as commonly done in power electronics. One of these designs uses the pidtune function and the other uses the sisotool, both from MATLAB. The proposed algorithm is based on an automatic tuning of the PID gains, guided by the minimization of an objective function that takes into account phase margin and crossover frequency, for a set of 4 plants. In addition, an extension for a more complex converter is presented, specifically a buck-boost converter used in small satellites, to show the suitability of the proposed robust control design for DC-DC converters. The fixed control gains designed here completely offline, with good convergence in the performed case studies, allowing better performances than those obtained by conventionally designed PID controllers.

\section{PRoblem Description}

The aim of this section is to illustrate two conventional PID designs and to describe the robust control problem to be addressed here. For sake of example, consider the output voltage control of a buck converter [14], where $L$ is the filter inductor, $C$ is the filter capacitor, $R_{L}$ is the resistive load, $V_{g}$ is the input voltage and $V_{o}$ is the output voltage of the converter, to be regulated.

The buck converter has the transfer function

$$
G_{p}(s)=\frac{R_{L} V_{g}}{s^{2}\left(C L R_{L}\right)+s L+R_{L}}
$$

considering the duty cycle as input variable and the capacitor voltage as the output variable [14].

Differently from similar results in the literature, here some parameters of this converter are assumed, in the control design stage, as uncertain. Specifically, consider that the value of the load resistor $R_{L}$ and the input voltage $V_{g}$ are assumed as not precisely known, but belonging to real intervals for which only the upper and lower bounds are known, as given in Table I.

Table I

BUCK PARAMETERS.

\begin{tabular}{c|c}
\hline Parameters & Values \\
\hline Input voltage $\left(V_{g}\right)$ & $30 \mathrm{~V} \pm 10 \%$ \\
Output voltage (on load) $\left(V_{o}\right)$ & $15 \mathrm{~V}$ \\
Switching frequency $\left(f_{s}\right)$ & $30000 \mathrm{~Hz}$ \\
Filter capacitor $(C)$ & $100 \mu \mathrm{F}$ \\
Filter inductor $(L)$ & $100 \mu \mathrm{H}$ \\
Resistive load $\left(R_{L}\right)$ & $3 \Omega \pm 50 \%$ \\
\hline
\end{tabular}

The control of the output voltage of the buck converter used here has a unit feedback, with the controller $G_{c}$ being a fixed gain PID controller given by

$$
G_{c}(s)=\left[\frac{K_{d} s^{2}+K_{p} s+K_{i}}{s}\right] \times\left[\frac{p b}{p b+s}\right]
$$

$K_{p}, K_{i}$ and $K_{d}$ are, respectively, the proportional, integral and derivative gains. $p b$ is an additional pole placed at a high frequency to reduce interactions with the lead-lag action, and also to ensure a causal transfer function for the PID.

\section{A. Conventional designs}

As usually done for power converters, the gains of the PID can be computed in order to ensure 60 degree phase margin and a crossover frequency one decade below the converter switching frequency [14]. Two solutions for this control design problem are given in the sequence, one based on the function pidtune and the other based on iterative design in sisotool, both relying on MATLAB.

Choosing the case of maximum values of $V_{g}$ and $R_{L}$ to define the nominal plant for conventional control design, due to the fact that this is the plant with smaller damping factor, and the specifications of phase margin and crossover frequency given above, the MATLAB function pidtune provides the PID

$$
G_{c}(s)=\frac{\left(1.89 \times 10^{-6} s^{2}+0.0226 s+64.9\right) 7.129 \times 10^{4}}{s\left(s+7.129 \times 10^{4}\right)}
$$

It is worth to mention that the pidtune returned an ideal PID, and then an additional pole was included one decade above the fastest zero of the controller to get the result in (3).

Now, as most commonly done by control engineers, using an interative design in sisotool, from MATLAB, considering the same nominal plant and the same design specifications used in pidtune example, one can get, by trial and error, the PID controller

$$
G_{c}(s)=\frac{\left(4.16 \times 10^{-6} s^{2}+0.0315 s+56.29\right) 6.283 \times 10^{4}}{s\left(s+6.283 \times 10^{4}\right)}
$$

Figure 1 shows the step response of the closed-loop system with both conventionally designed controllers. One can see that the responses are quite similar, with a slight superior transient response from the sisotool based design.

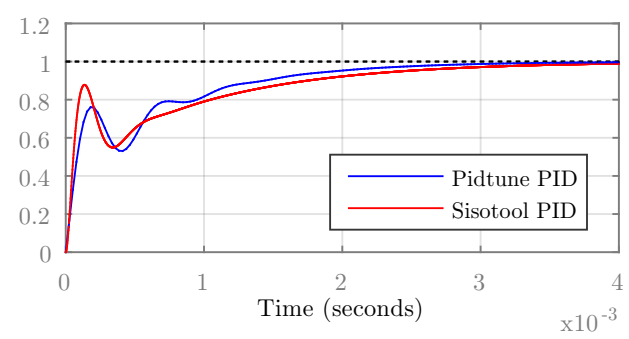

Figure 1. Converter with the MATLAB sisotool PID controller compared with the converter with the pidtune controller: Closed-loop system unit step response.

But the most important point to be remarked here is that these conventional designs need one nominal model of the plant to initiate the control design and, only a posteriori, the 
robustness and performance to intervalar parametric uncertainties are addressed. This can lead to a time consuming control design, based on trial and error sessions of a control engineer, which represents cost in industry.

This motivates the investigation in this paper, that aims to provide an automated tuning procedure for robust PID controllers, as described in the sequence.

\section{B. Control problem definition}

Find, by means of an automated tuning performed off-line, fixed gains $K_{p}, K_{I}$ and $K_{d}$, and also the additional pole $p b$ (i.e. four control parameters) for the PID in (2) such that, for all the extreme values of the plant model, the phase margin and the crossover frequency approach values prescribed by the control designer.

It is worth to mention that the controller solving the above design problem is a simple fixed gain PID, thus avoiding more complex implementations of PIDs, based on strategies that lead to time-varying control gains. Such controller, obtained in an off-line procedure can be useful as a benchmark for comparisons with other controllers.

\section{Proposed Design Solution}

In order to provide a solution for the above design problem, consider the vector of control parameters given by

$$
K=\left[\begin{array}{llll}
K_{p} & K_{I} & K_{d} & p b
\end{array}\right]
$$

To measure stability and the performance of the control system with these gains when affected by uncertain parameters, the following function are used here

$$
\begin{aligned}
f_{j}(K)=\delta_{j}(K)\left[\left|\left(P M^{\star}-P M_{j}(K)\right)\right| \gamma_{1}\right. \\
\left.+\left|\left(\omega_{c}^{\star}-\omega_{c j}(K)\right)\right| \gamma_{2}\right], j=1, \ldots, p
\end{aligned}
$$

where $\mathrm{PM}^{\star}$ and $\omega_{c}^{\star}$ are reference values for desired phase margin and crossover frequency, respectively.

This function provide, for each one of the $p$ possible conditions of extreme plant uncertain parameters, a weighted sum of the deviations of the phase margin and of the crossover frequency to their desired values.

The weights $\gamma_{1}$ and $\gamma_{2}$ are chosen, for instance, to compatibilize the size of the parcels in functions (6) or to set a higher importance for one of the perfomance indices (phase margin and crossover frequency) over the other. For each $j, \delta_{j}(K)$ is a scalar that plays the role of a penalty factor, being set to a high value when the control gain $K$ under test produces instability, or being set to 1 when no unstable closed-loop pole is detected for any of the parameter situations $j=1, \ldots, p$.

Notice that, for each control gain $K$ under test, the values of $\mathrm{PM}_{j}(K)$ and $w_{c j}(K)$ can be easily obtained, for instance, from function margin, of MATLAB, and the closed-loop poles can also be obtained from the function pole, of MATLAB, thus allowing to decide for the high or unit value for $\delta_{j}(K)$.

Defining the objective function

$$
J(K)=\max \left\{f_{j}(K)\right\}, j=1, \ldots, p
$$

as the worst case value of the functions in (6), one has that the PID control gain vector that minimizes $J$ in a given search space $\mathcal{K}$ (i.e. a given subset of $\mathcal{R}^{4}$ ), is optimal in the sense of ensuring the smallest weighted sum of deviations from the desired values of phase margin and crossover frequency in this space of controllers. In other words

$$
K^{\star}=\arg \min _{K \in \mathcal{K}} J(K)
$$

Notice that $K^{\star}$ that solves (8) will also provide a solution for the practical control problem in Section II.B.

A mathematical solution for the optimization control problem (8) is difficult to be obtained, due to the difficulty in expressing the gradient of the objective function and due to possible discontinuities of the objective function in a given broad search space. Particle swarm optimization has proven to be efficient to find minimum vaues of nonlinear objective functions, in search spaces with possible discontinuities and with possible multiple local minima [30].

\section{A. Particle swarm optimization}

PSO is a bio-inspired algorithm based on intelligent swarms, where the collective behavior of locally-interacting nonsophisticated agents creates global functional patterns, proposed by J. Kennedy and R. C. Eberhart in [30].

For the search of control gains using the PSO, the PID (9) can be seen as a particle $i$ in the search space, that is, each $K$ is associated with the position

$$
s_{i}=\left[\begin{array}{llll}
K_{p i} & K_{I i} & K_{d i} & p b_{i}
\end{array}\right]
$$

The particle swarm has a size of $N$ particles, with $N$ chosen sufficienly large to cover the search space.

The particles will evolve in the search space, that is, their positions will be updated from one epoch $k$ to the next epoch $k+1$, until reaching the limit of epochs $M$ for evolution.

Thus, at the epoch $k$, each particle has a position $s_{i}(k)$ and its own velocity $v_{i}(k)$, moving through the search space governed by the equations

$$
\begin{gathered}
s_{i}(k+1)=s_{i}(k)+v_{i}(k+1) \\
v_{i}(k+1)=\omega v_{i}(k)+\phi_{1}\left(\operatorname{rand}_{1}\left(P_{i . b e s t}-s_{i}(k)\right)\right) \\
+\phi_{2}\left(\operatorname{rand}_{2}\left(G_{\text {best }}-s_{i}(k)\right)\right)
\end{gathered}
$$

Each particle memorizes the position that got its best fitness, called $P_{i . b e s t}$, and the swarm is also influenced by the particle that obtained the best fitness position among all particles, called $G_{b e s t}$. This fitness is defined here by the objective function (7). $\phi_{1}$ and $\phi_{2}$ are the cognitive and social coefficients, respectively, $\omega$ the inertia and $\operatorname{rand}_{1}$ and $\operatorname{rand}_{2}$ random values between $[0,1]$. It is also noted that the position and velocity of each particle are represented by vectors [31].

To guide the choice of the PSO parameters, the number of particles and of epochs, respectively, $N$ and $M$, and the coefficients $\phi_{1}$ and $\phi_{2}$ are chosen in order to ensure convergence of the fitness function with low computational effort. 
Finally, in practical terms, it is worth to mention that one solution of the control problem in Section II can be achieved by the control designer choosing the worst case plant as the one more difficult to be stabilized (e.g. the one with smaller damping ratio or the one with smaller bandwidth). Then, a PID can be designed with a conventional technique, as the pidtune, exemplified in Section II. The stability and the performance of this controller with the other plant situations can be, a posteriori, checked. This strategy can lead to acceptable performance but there is no guarantee that better performances could not be found in a search space including this conventionally designed PID. If a search space is stablished around this viable controller, exhaustive grids would become rapidly prohibitive in terms of computational time. For instance, a simple cube in the search space, with side 1, discretized in 1000 points each side, would demand the test of $10^{6}$ controllers. The proposed solution with PSO allows to highly improve the results obtained with conventional designs, as will be illustrated in the next section.

\section{PSO APPLIED TO THE BUCK CONVERTER CASE STUDY}

In Section II, conventional PIDs were designed for a buck converter, using only one nominal model of the plant in the design. Now consider the same set of parameters given in Table I, for the design of a robust PID with the help of the PSO described in the previous section.

The design specifications remain the same: $\mathrm{PM}^{\star}=60^{\circ}$ and $\omega_{c}^{\star}=3000 \mathrm{~Hz}$, that is, one decade below the converter switching frequency. The weights in the terms of the objective function (6), which will guide the PSO, are $\gamma_{1}=1$ and $\gamma_{2}=10^{-2}$, and $\delta_{j}(K)$ is $10^{3}$ in case of instability or 1 in the case of stability.

The search space chosen here is a hyper-rectangle around the parameters of the PID obtained from the pidtune function, given in (3), since this controller can be easily reproduced. The search space is described as $2.26 \times 10^{-5} \leq K_{p} \leq 22.6$, $6.49 \leq K_{I} \leq 6.49 \times 10^{2}, 1.89 \times 10^{-9} \leq K_{d} \leq 1.89 \times 10^{-3}$ and, to keep the additional pole in high frequency range, $0.5 \times$ $7.1 \times 10^{4} \leq p b \leq 1.5 \times 7.1 \times 10^{4}$. Even though parts of the search space represent PID parameters which will produce instability, the PSO will penalize these individuals with the high value of $\delta_{j}(K)$ in (6).

The algorithm starts with the following parameters: swarm size $N=100$, maximum number of iterations (epochs) $M=$ 50 and social and coefficient $\phi_{1}$ and $\phi_{2}$ with values of 0.5 each one. A random population of particles is initialized and then the PSO algorithm updates their position and velocity, based on (10) and (11), and evolves until the maximum number of epochs is reached or the stall criterion of 30 epochs without significant reduction in the objective function. The final value of the objective function obtained in one of the executions of the PSO is $J(K)=11.06$, with the evolution of the fitness illustrated in Figure 2 (a).

The best particle found by the PSO, that is, the best PID control parameter vector with respect to the minimization of the objective function (7), is given by

$$
G_{c}(s)=\frac{\left(5.31 \times 10^{-6} s^{2}+0.0571 s+292.8\right) 8.266 \times 10^{4}}{s\left(s+8.266 \times 10^{4}\right)}
$$

For a performance comparison, the MATLAB step responses shown for the conventional controllers in Section II are reproduced now together with the response of the robust PID tuned by the PSO, in Figure 2 (b), where one can see the clear superiority of the performance with the controller obtained with the proposed method.
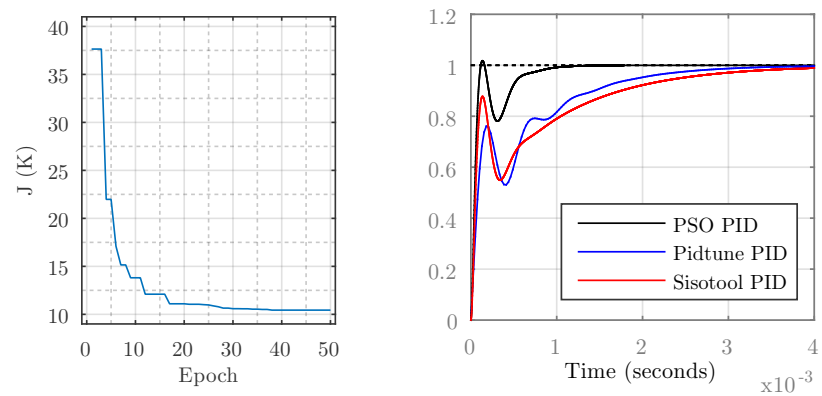

Figure 2. (a) Best fitness value in each epoch. (b) Comparison of the closedloop system unit step response with the conventionally designed PIDs (3) and (4) and with the PSO (12).

To have a more realistic analysis of the PID controllers, comparisons of the other two conventionally designed PIDs obtained in Section II with the robust PID from PSO are carried out, based on the software PSIM with ideal components, including the effect of the PWM signal as input of the filter and load stage. Figure 3 (a) presents the startup transient response with the plant with maximum values of $R_{L}$ and $V_{g}$, which is the situation used in the design of the conventional PID controllers. One can confirm the superiority of performance with the controller obtained with PID designed with the help of PSO.

For the result in Figure 3 (b), one has the system operating with each one of the PIDs, in steady state, in the mean parameter situation, and then the input voltage $V_{g}$ is reduced in $10 \%$. Again, one can see the superior transient recover from the PID tuned by the PSO. The result in Figure 3 (c) has the same initial conditions of the previous test, and a sudden reduction of $50 \%$ in $R_{L}$ is applied (i.e. the load power consumption is suddenly increased). Again, the superior performance with the PSO based PID controller is confirmed.

To confirm robustness against parameter uncertainty, Figure 4 (a) shows the startup transient response of the closedloop system with the PID tuned by the proposed PSO, for all the extreme load situation conditions. The line in blue represents the case of the plant with mean parameter values, and the lines in black represents the converter with the upper and lower bounds of $R_{L}$ and $V_{g}$. It is noticed that all responses show good transient performance, due to the guaranteed phase margin and crossover frequency. 

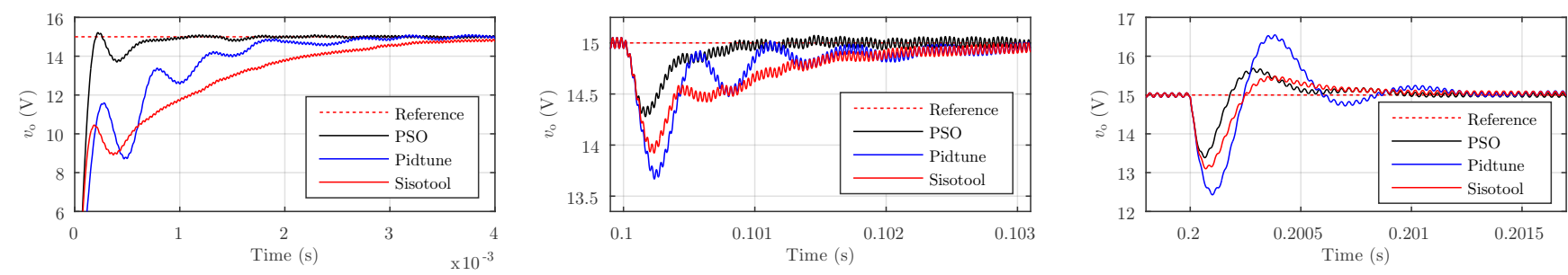

Figure 3. PSIM simulation of the closed-loop system for the converter with upper bounds of $R_{L}$ and $V_{g}$ : (a) System start-up. (b) $10 \%$ reduction in input voltage $V_{g}$ in $t=0.1 \mathrm{~s}$. (c) $50 \%$ reduction in load resistance $R_{L}$ in $t=0.2 \mathrm{~s}$.
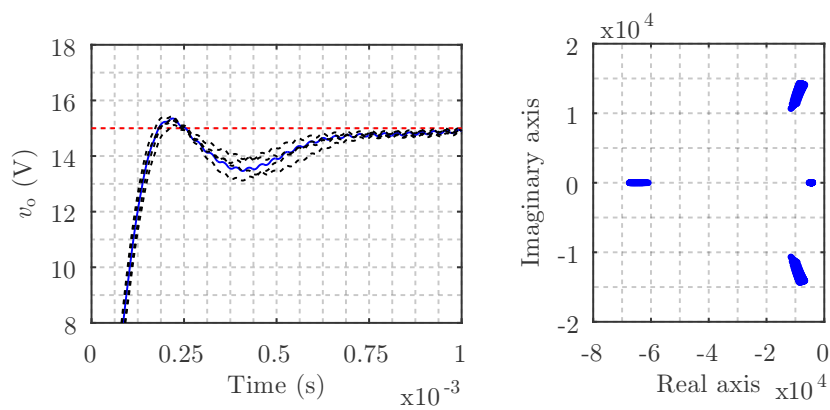

Figure 4. (a) Closed-loop start-up transient response system with nominal transfer function (in blue) and the upper and lower bounds of $R_{L}$ and $V_{g}$ (in black). (b) Closed-loop pole location.

Finally, Figure 4 (b) corroborates the stability of the system with the PID tuned with the help of PSO, for the domain of uncertain paramters, showing that all closed-loop poles remain on the lefthand side of the complex plane.

\section{EXTENSION: BUCK-BOOST FOR SMALL SATELLITES}

To apply the proposed control design to a more complex case, consider the converter in Figure 5, used in small satellites, which performs distributed bus power processing [32]. This converter operates in two different ways, which vary depending on the evolution of the satellite orbit. In the eclipse period when there is no solar energy in the photovoltaic panels, the converter regulates the output bus for the loads. In the sunlight period, when the photovoltaic panels are receiving solar radiation, the converter operates as a battery charger. For this example it was used only the eclipse converter plant, given by equation (13), which is represented by the averaged small signal model [33]-[35]

$$
G_{v_{o} d}(s)=\frac{a_{2} s^{2}+a_{1} s+a_{o}}{b_{3} s^{3}+b_{2} s^{2}+b_{1} s+b_{o}}
$$

being

$a_{0}=R_{b}-R_{o}-D^{2} R_{o}+2 D R_{o} ; a_{1}=L-C_{b} R_{b} R_{o}-$ $C_{b} D^{2} R_{b} R_{o}-C_{i} D^{2} R_{b} R_{o}+2 C_{b} D R_{b} R_{o}+C_{i} D R_{b} R_{o}$; $a_{2}=C_{b} L R_{b}+C_{i} L R_{b} ; b_{o}=R_{b}+R_{o}+D^{2} R_{o}-2 D R_{o}$; $b_{1}=L+C_{b} R_{b} R_{o}+C_{b} D^{2} R_{b} R_{o}+C_{i} D^{2} R_{b} R_{o}-2 C_{b} D R_{b} R_{o}$; $b_{2}=C_{b} L R_{b}+C_{i} L R_{b}+C_{i} L R_{o} ; b_{3}=C_{b} C_{i} L R_{b} R_{o}$

For sake of comparison, a PID controller was designed with pidtune to achieve a phase margin of $60^{\circ}$ and a crossover

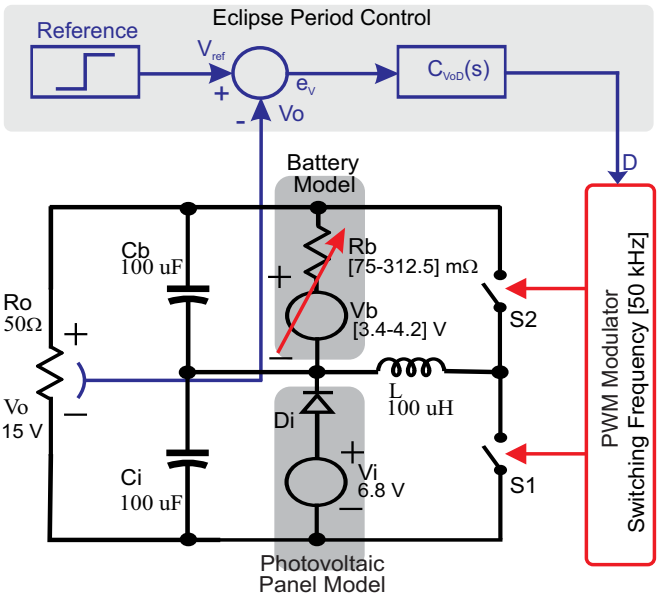

Figure 5. Bidirectional buck-boost converter with stacked input and output for small satellite application.

frequency of $400 \mathrm{~Hz}$, and these requirements were also used to guide the design with the PSO, with the same parameters employed in Section IV (swarm size and number of iterations), leading to the controller

$$
C_{v_{o} d}(s)=\frac{\left(4.95 \times 10^{-6} s^{2}+0.0045 s+38.67\right) 4.755 \times 10^{4}}{s\left(s+4.755 \times 10^{4}\right)}
$$

Figure 6 shows the step responses for this controller and for the PID designed with pidtune, under the same design requirements. One can see the superiority of the PID obtained from the proposed procedure, providing the faster responses in Figure 6 (two upper curves), also for a more challenging application.

\section{CONCLUSION}

This paper provided an alternative for automated design of robust (fixed gains) PIDs with application for power converters with uncertain parameters. The proposed solution relies on a PSO that minimizes an weighted sum of the deviations of desired values for phase margin and crossover frequency, allowing results superior than conventionally designed fixed gains PIDs. The main advantage of the proposed design procedure is to alleviate the control engineer from the time consuming task of obtaining the control gains by trial and error sessions. This task is delegated to an automated design 

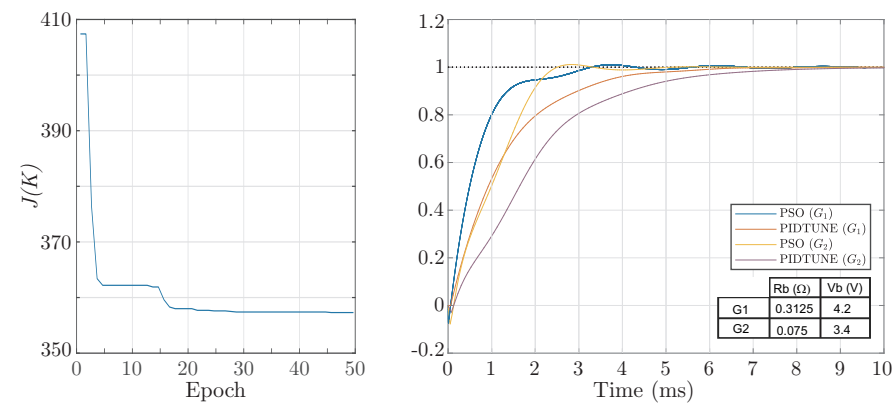

Figure 6. Step responses for the PID obtained from pidtune function and for the proposed PID in (14).

using PSO, which produce, in an offline procedure, the gains of robust PIDs, suitable for control of power converters.

\section{REFERENCES}

[1] S. Bhattacharyya, "Robust control under parametric uncertainty: An overview and recent results," Annual Reviews in Control, vol. 44, pp. 45-77, 2017.

[2] K. J. Åström, T. Hägglund, C. C. Hang, and W. K. Ho, "Automatic tuning and adaptation for PID controllers - a survey," Control Engineering Practice, vol. 1, no. 4, pp. 699-714, 1993.

[3] H. Hjalmarsson, M. Gevers, S. Gunnarsson, and O. Lequin, "Iterative feedback tuning: theory and applications," IEEE control systems magazine, vol. 18, no. 4, pp. 26-41, 1998.

[4] K. J. Astrom, T. Hagglund, and K. J. Astrom, Advanced PID control. ISA-The Instrumentation, Systems, and Automation Society Research Triangle ..., 2006, vol. 461.

[5] M.-T. Ho and C.-Y. Lin, "PID controller design for robust performance," IEEE Transactions on Automatic Control, vol. 48, no. 8, pp. 1404-1409, 2003.

[6] G. Feng, E. Meyer, and Y.-F. Liu, "A new digital control algorithm to achieve optimal dynamic performance in DC-to-DC converters," IEEE Transactions on Power Electronics, vol. 22, no. 4, pp. 1489-1498, 2007.

[7] J. G. Ziegler and N. B. Nichols, "Optimum settings for automatic controllers," trans. ASME, vol. 64, no. 11, 1942.

[8] Z.-Y. Zhao, M. Tomizuka, and S. Isaka, "Fuzzy gain scheduling of PID controllers," IEEE transactions on systems, man, and cybernetics, vol. 23, no. 5, pp. 1392-1398, 1993.

[9] R. K. Mudi and N. R. Pal, "A robust self-tuning scheme for PI- and PD-type fuzzy controllers," IEEE Transactions on fuzzy systems, vol. 7, no. 1, pp. 2-16, 1999.

[10] L. Guo, J. Y. Hung, and R. M. Nelms, "Evaluation of DSP-based PID and fuzzy controllers for DC-DC converters," IEEE transactions on industrial electronics, vol. 56, no. 6, pp. 2237-2248, 2009.

[11] M. Algreer, M. Armstrong, and D. Giaouris, "Adaptive PD+I control of a switch mode DC-DC power converter using a recursive fir predictor," 2010.

[12] H.-X. Li, L. Zhang, K.-Y. Cai, and G. Chen, "An improved robust fuzzyPID controller with optimal fuzzy reasoning," IEEE Transactions on Systems, Man, and Cybernetics, Part B (Cybernetics), vol. 35, no. 6, pp. 1283-1294, 2005.

[13] W. K. Ho, C. C. Hang, and J. Zhou, "Self-tuning PID control of a plant with under-damped response with specifications on gain and phase margins," IEEE Transactions on Control Systems Technology, vol. 5, no. 4, pp. 446-452, 1997.

[14] R. W. Erickson and D. Maksimovic, Fundamentals of power electronics. Springer Science and Business Media, 2007.

[15] L. Guo, J. Y. Hung, and R. Nelms, "PID controller modifications to improve steady-state performance of digital controllers for buck and boost converters," in APEC. Seventeenth Annual IEEE Applied Power Electronics Conference and Exposition (Cat. No. O2CH37335), vol. 1. IEEE, 2002, pp. 381-388.

[16] V. Yousefzadeh, A. Babazadeh, B. Ramachandran, E. Alarcón, L. Pao, and D. Maksimovic, "Proximate time-optimal digital control for synchronous buck DC-DC converters," IEEE Transactions on Power Electronics, vol. 23, no. 4, pp. 2018-2026, 2008.
[17] S. Kapat and P. T. Krein, "Formulation of PID control for DC-DC converters based on capacitor current: A geometric context," IEEE Transactions on Power Electronics, vol. 27, no. 3, pp. 1424-1432, 2011.

[18] H.-H. Park and G.-H. Cho, "A DC-DC converter for a fully integrated PID compensator with a single capacitor," IEEE Transactions on Circuits and Systems II: Express Briefs, vol. 61, no. 8, pp. 629-633, 2014.

[19] E. W. Zurita-Bustamante, J. Linares-Flores, E. Guzman-Ramirez, and H. Sira-Ramirez, "A comparison between the GPI and PID controllers for the stabilization of a DC-DC "buck" converter: A field programmable gate array implementation," IEEE Transactions on Industrial Electronics, vol. 58, no. 11, pp. 5251-5262, 2011.

[20] C. Chang, Y. Yuan, T. Jiang, and Z. Zhou, "Field programmable gate array implementation of a single-input fuzzy proportional-integralderivative controller for DC-DC buck converters," IET Power Electronics, vol. 9, no. 6, pp. 1259-1266, 2016.

[21] U. A. Shaikh, M. K. AlGhamdi, and H. A. AlZaher, "Novel product ANFIS-PID hybrid controller for buck converters," The Journal of Engineering, vol. 2018, no. 8, pp. 730-734, 2018.

[22] S. E. De León-Aldaco, H. Calleja, and J. A. Alquicira, "Metaheuristic optimization methods applied to power converters: A review," IEEE Transactions on Power Electronics, vol. 30, no. 12, pp. 6791-6803, 2015.

[23] C.-L. Lin, H.-Y. Jan, and N.-C. Shieh, "GA-based multiobjective PID control for a linear brushless DC motor," IEEE/ASME transactions on mechatronics, vol. 8, no. 1, pp. 56-65, 2003.

[24] Z.-L. Gaing, "A particle swarm optimization approach for optimum design of PID controller in AVR system," IEEE transactions on energy conversion, vol. 19, no. 2, pp. 384-391, 2004.

[25] R. A. Hanifah, S. F. Toha, S. Ahmad, and M. K. Hassan, "Swarmintelligence tuned current reduction for power-assisted steering control in electric vehicles," IEEE Transactions on Industrial Electronics, vol. 65 , no. 9, pp. 7202-7210, 2017.

[26] J. Darvill, A. Tisan, and M. Cirstea, "A novel PSIM and matlab cosimulation approach to particle swarm optimization tuning of PID controllers," in 2014 International Conference on Optimization of Electrical and Electronic Equipment (OPTIM). IEEE, 2014, pp. 784-789.

[27] E. Sahin, M. S. Ayas, and I. H. Altas, "A PSO optimized fractional-order PID controller for a PV system with DC-DC boost converter," in 2014 16th International Power Electronics and Motion Control Conference and Exposition. IEEE, 2014, pp. 477-481.

[28] P. Farhang, A. M. Drimus, and S. Mátéfi-Tempfli, "New technique for voltage tracking control of a boost converter based on the PSO algorithm and LTspice," in 2015 56th International Scientific Conference on Power and Electrical Engineering of Riga Technical University (RTUCON). IEEE, 2015, pp. 1-6.

[29] P. Verma, N. Patel, N.-K. C. Nair, and A. Sikander, "Design of PID controller using cuckoo search algorithm for buck-boost converter of LED driver circuit," in 2016 IEEE 2nd Annual Southern Power Electronics Conference (SPEC). IEEE, 2016, pp. 1-4.

[30] R. Eberhart and J. Kennedy, "A new optimizer using particle swarm theory," in In Proceedings of the Sixth International Symposium on Micro Machine and Human Science. IEEE, 1995, pp. 39-43.

[31] M. Veerachary and A. R. Saxena, "Optimized power stage design of low source current ripple fourth-order boost dc-dc converter: A pso approach," IEEE Transactions on Industrial Electronics, vol. 62, no. 3, pp. 1491-1502, 2015.

[32] E. Mattos, A. M. Andrade, G. V. Hollweg, M. L. d. S. Martins, and J. R. Pinheiro, "Analysis and design of a stacked power subsystem on a picosatellite," IEEE Aerospace and Electronic Systems Magazine, vol. 33, no. 10, pp. 4-13, 2018.

[33] D. DePasquale and J. Bradford, "Nano/microsatellite market assessment," Public Release, Revision A, SpaceWorks, 2013.

[34] R. Burt, "Distributed electrical power system in cubesat applications," 2011.

[35] T. J. Doering, "Development of a reusable cubesat satellite bus architecture for the kysat-1 spacecraft," 2009. 